Euskal ikerketen aldizkaria | Revue d'études basques |

Revista de estudios vascos | Basque studies review

$6 \mid 2001$

Numéro VI

\title{
Jean-Martin Hiribarren, Iruñeko bestak 1845
}

\section{Patri Urkizu}

\section{OpenEdition \\ Journals}

Édition électronique

URL : http://journals.openedition.org/lapurdum/1236

DOI : 10.4000/lapurdum.1236

ISSN : 1965-0655

Éditeur

IKER

Édition imprimée

Date de publication : 1 octobre 2001

Pagination : 319-351

ISBN : 2-84127-156-0

ISSN : $1273-3830$

Référence électronique

Patri Urkizu, « Jean-Martin Hiribarren, Iruñeko bestak 1845 », Lapurdum [Linean], 6 | 2001, Sarean emana----an 01 juin 2009, kontsultatu 31 janvier 2020. URL : http://journals.openedition.org/ lapurdum/1236 ; DOI : 10.4000/lapurdum.1236 


\section{Patri Urkizu}

(UNED)

\section{Jean-Martin Hiribarren, Imuñeko bestak 1845}

Agosti Chaho, Jean Duvoisin, Maurice Harriet, Joanes Oxalde, Emmanuel Inchauspe, Jean-Martin Hiribarren... Hona 1810etik 15erako epean Euskal Herriko Iparraldean jaiotako euskaltzale ospetsuen zenbait izen. Lore-xorta ederra benetan! Euskal hizkuntza eta literaturaren pizkundean zuzi distiratsu eta itzalezinak, Pierre Lafittek' ongiaski azpimarratzen digun arabera.

Azkainen sortua genuen Hiribarren, etorki nobleko familia batean, Etcheberria baitan, hain zuzen. Bere amona Juana Dutari, Dominike de Hiribarrenen emaztea, Baztango Dutari Azpilikuetarren leinu famatutik zetorren. Honetakoak izan ziren, ere, besteak beste, San Frantzisko Xabier eta Martin Azpilikueta 'doctor navarrus' ospetsuak.

Hona bere aitaren heriotze biharamunean besteren artean idatzi zuen bertso xorta gorabehera zenbait aipatuz:

Guziek bazakiten zenbat zen zuzena,

Hauzi guzietan zen lekhuko emana,

Frantziako aferak nahasi zirenian

Ihesari eman zen denbora zuenian.

Aita amak ziozten gathibo egorri,

Frantziarat norapeit bihotz zilhagarri,

Berak jo zuen urrun Madrilgo alderat,

Gorthian gora zituan ahaiden arterat,

Haren aitatxia aiphatu Duthary,

Espainiako gorthian lehen aitzindari².

\footnotetext{
1 Pierre Lafitte, "Jean-Martin Hiribarren (1810-1866)", Euskal Herria (I 789-1850). Actes du colloque international d'Etudes Basques (Bordeaux 3-5 mai 1973), Société des Amis du Musée Basque. Bayonne, 1978, 181-191

2 Patri Urkizu, "Euskal idazleen bertsoak bigarren Errepublikaren gainean (1848-1851)", "La révolution française dans t'histoire et la littérature basques du XIXe siècle". Sous la direction de JeanBaptisti Orpustan, Izpegi, Baigorri, 1994, 121-147.
} 
Hamalau urte zituela joan zen apaiz ikasketak Baionako seminarioan burutzera eta bertan apeztu zen 1833. ekainaren batean, hogeita hiru urte zituelarik. Denboraldi bat Urruñan eman ondoren Goyetche erretoraren laguntzaile, Bardozera iragan zen apez-laguntzaile gisara, eta apez nagusi bilakatu Borda 1839an hil zenean, bertako arimen gidaritza 1865. urterarte hartuz. Nekaturik, Baionako ohorezko kalonje izendatu zuten, baina berehala kota gaitzak jota hurrengo urtean hil zen.

Langile amorratu eta apala, bere eleizkizunek eta gazteendako ardurak uzten zizkioten uneak oro idazten murgildurik igarotzen zituen, eta bizi zelarik argia ikusi zuten poemen artean hauexek dauzkagu : Iruñeco bestak.3 (1845); Montebideoco berriac ${ }^{4}$ (1853); Eskaldunac 5 ... (1853).

Montebideoco berriac maiatzean plazaratu zen. Poemak 223 lauko ditu, hamahiru silaba duelarik bertsolerro bakoitzak eta binaka errimak bildurik. Euskal Herriko odoluste izugarri haren aurka jasotako ahotsa izan zen, eta irailean Abbadiak antolatu lore Jokoetan ere gaia beraxe izan zen.

Eskaldunac, abenduan argitaratua, oraindik askoz ere luzeagoa da, bost mila bertsolerro inguru baitu, Euskal Herrietako historia egiten du bertan hala nola bertan sorturiko hainbat eta hainbat pertsonaia garrantzizkoeren bizitza zertzeladak ematen. Hona zioena Andima Ibiñagabeitiak ${ }^{6}$ obra honetaz :

...olerkari baino neurtizlariago ageri zaigu Hiribarren. Alare ba ditu bere bertsu aldietan poeta garaienak ere onartuko lituken ahapaldiak. Arrigarria benetan apaiz onez euskera nola menderatu eta erabiltzen zuen ikustea, txoriari tzinta bezin errex ta aisa ateratzen zaizkio bertsuak lumamuturretik.

\footnotetext{
${ }^{3}$ Ariel aldizkaria. Baiona, $\mathrm{n}^{\circ} 53,54,55,56,57,59,60,61 ; 1845-\mathrm{X}-5,12,19,26-, \mathrm{X} \mathrm{I}-2,16,23,30$. Bukatugabea.

${ }^{4}$ Montebideoko berriac. Bayonne, Foré et Lasserre, 1853. Patri Urkizu (ed., 1991), Lapurdi, Baxanabarre era Zuberwako Bertso eta Kantak. II, 1545-1900, 543-584.

5. Eskaldunac, Iberia, Cantabria, Eskal-herriac, Eskal-Herri bakhotcha eta hari darraicona. Foré eta Laserre imprimerian. Baionan, 1853. Fac-simil, Enciclopedia General Ilustrada del País Vasco, Zarautz, 1971.

6 Andima Ibiñagabeitia, "Hiribarren'en "Eskaldunac" (1853-1953)", Gernika, n'22, Paris, 1953, 143.
} 
Harmada

\section{3}

Ederra zen egun hetan,

Iruñeko zelhaietan,

Soldadu lerruan emana,

Francisco Asisek ${ }^{59}$ mana.

Beruak etzuen izitu

Nahiz nola zen agertu

Gerla bide itzulitan

Utziak aski berritan.

164

Jendia zagolarik erria,

Sapha minian baitzen airia,

Soldaduak arratsaldian

Kausten ziren hoberenian;

Ibili ziren lasterka,

Aitzindariak oihuka,

Goiti behiti lerrotan,

Erregen gustu handitan.

165

Sarthu zirenian hirian

Etzen bustirik bisaian;

Hanbat lasterken ondotik,

Doian ezagun hatsetik

Asisen zaldizkuek zuten

Sartzian ederren ematen;

Bainan bazen oinezkotan

Zain hartagorik menbrotan.

166

Halako soldaduekin,

Aitzindari jakinekin,

Bentzu diteke mundua,

Nahi bezin hedatua.

Ez dire beldur gosiaren,

Ez eta ere bidiaren,

Guti janik ikhasiak

Laster higaten mendiak.
167

Suntsuna, xirribikekin,

Nafartar soldaduekin,

Gerian dabilke jostetan

Nola Eliza bestetan.

Goizeti arrats askotan

Suan eta mendietan,

Zigarroa bat musuan

Bizi ditezke multzuan.

168

Sos baten agorienta

Hekin edari plazenta;

Soldadu on ilkhitzeko

Bada heki beiratzeko.

Erromanuen denboretan

Lehoin ziren mendietan;

Ondoriuak bizi dire

Gutiago ez baitire.

169

Bertze hanbat jende zoro,

Guziak direnian haro,

Zer diren eskaldun umiak

Errateko zen, aizkidiak :

Lau mila urthe bederen

Nausi dire toki beren;

Ez da munduan bertzerik

Hek bezala itzaturik.

170

Higatu dire mendetan

Populuak bazterretan;

Bere lurretik suntsitu,

Nausiek asko kasatu,

Eskalduna Pirenetan

Ez da izitu gerletan

Aitak ehortzi tokian

Izanen bethi agian.

AskiJ.M.H.

59 Francisco de Asis Maria Fernando de Borbón (Aranjuez, 1822 - Epinay-sur-Seine, 1902). i 846ean bere lehengusina Isabel IIarekin ezkonduzen 
berritzeko hauteskundeak prestatu zituzten. Askatasunaren zuhaitza plaza erdian jaso zuten eta okasio honekin Hiribarrenek bandera errepublikanoa bedeinkatu zuen, ondoren frantsesez solas eder batez mintzatu zelarik askatasuna (Libertatea zein eder den!-errepikatuz euskaraz-), berdintasuna eta anaitasuna goraipatuz. Lafittek zati bat ematen digularik honako hitzak eransten dizkio, je me demande si le discours de Hiribarren n'est pas un chefd'oeuvre de ce genre bâtard, alegia, jenero bastart honetan maisu lantzat jo daitekeela. Baina, gauza jakina den bezala, zirkunstantziazko literatura hori ugaria da herrialde guztietako literaturetan.

\section{IRUÑEKO BESTAK 1845}

Hiribarren berak dioenez, lapurteraz idatzitako poema txiki bat da "Petit poème sur les fêtes de Pampelune 1845. En dialecte labourdin le plus pur". Baditu, ordea, poematxo honek ehun eta hogeitamar zortziko, beraz, 1362 bertsolerro, aitzakitzat harturik Chahok, Nemours-eko duke eta dukesa, Aumale-ko dukeak eta beste konpainia eder batek (tartean bera) 1845 urteko irailean Iruñeara egin zuten bidaia eta hemen dastatu zituzten bestak.

Dukeen Euskal Herrirako bisitak utzi zizkigum hainbat bertso eta kanta nola Bizente Etxegaraik ${ }^{14}$ eskaini ziena Donostiatik igarotzean, edota Tolosatik ${ }^{15}$, edota Hazparneko Larralde Bordaxurik ${ }^{16}$ kantatuak. Baina ez ziren iristen Hiribarrenen tamainara, noski, zeinen obraren zati bat Chahok argitaratu zuen, pentsatzen baitzuen edizio elebiduna, euskara-frantsesa, egitea, asmo huts gelditu zena, ordea, Lafittek dioskun legez.

Azkaindarrak atharraztarraren aipamenarekin hasten digu poema Arielen, kortesiazko deikia, noski, baina poema berridatzi zuenean, urteak igaro eta, zati hau aldatu egin zuen, beste zenbait orraztu eta zuzenduz. Beraz azken idazkera moldatu dugu gure ediziorako. Bidaiariak, muga pasa ondoren Urdazubi, Arizkun, Elizondo, Moringo Zubia, Belate, Ultzama eta azkenean Iruñeara iritsiko dira erregina Isabel Ilarekin eta honen gortearekin elkartzeko.

Hau bere ama Kristina eta ahizpa Luisa Femandarekin Donostiatik zetorren, hemen mainu batzuk hartu eta hiriak harrera benetan ederra eskaini ondoren. Boulevard-eko Txakur-Txulo zeritzaion ostatuan egonak ziren eta musika jaialdi bat oparitu zion hiriak Luisa Fernandari, Bizente Etxegaraik,

14 Le Phare des Pyrénées, zb. 729,1845. VIII.13; zb. 732,1845.VIII.20.

15 Idem, zb. 740, 1845.IX.7; Le Courrier de la Gironde, 1845.IX.8

16 Id., zb. 744, 1845. IX. 17. 
okasio guztietarako prest zegoen donostiar poetak asmatu zortzikoak kantatu zizkiotelarik. Hauetariko bigarrenak honela zioen :

Infanta gaztetxoa
Espainiakoa,
Isabelen aizpatxo
biotz gurekoa:
erregeren alaba
zorionekoa,
ar ezazu oroitze
Donostiakoa 17.

Hemendik Iruñeara joan ziren eta Joakin Ignazio Menkos, Guendulaingo kontearen etxean hartu zuten ostatu. Eta bertan biltzen ziren Frantziako printzeekin, egun batean hirurogeita hamar gonbitatuei platera ugari eta onaskoak eta dantza eder bat eskaini zitzaielarikı1.

Poemak ez dion arren, printze frantsesek Ezpeletako Kontearen jauregian hartu zuten ostatu, bertan banketeen ondoren serenatak, gau-dantza eta gau-argiak dastatu zituztelarik. Erregina Isabelek Frantziako printzeak erregalatu nahi zituen, eta honetarako momentuan zen toreatzailerik famatuenari eskatu zion torea zezan Iruñean. Gonbitea Ministro de Instrucción y de Hacienda zenak berak egin zion, eta Madridetik pasatzerakoan Montes bere koadrilarekin jai eder bat ere eskaini zitzaion.

Frantziako eta Espainiako korteak Iruñean, iraun zuten bitartean, hau jendez beterik zegoen, ezen topaketa ilustrearen aitzakiaz iragarritako ikuskizunek Pirineoetako mainutegietarik ur-hartzaile anitz erakarri baitzuten.

Poemak bederatzi atal ditu : Bidaia (1-12); Argiak (13-2l); Zezenetako tokia (22-62); Zezenak (63-125); Zaldi Lasterketak (126-140); Zuziak (141-148), Zuziazko Tiruak (149-155); Pasaiuak (156-162); eta Harmada (163-170).

Zatirik nagusienak, beraz, zezen plazako giroak eta zezenek hartzen dute (22b.-125b.), bertan kantatzen direlarik garaiko toreadore famatuenaren,

\footnotetext{
17 Jose Vicente de Echegaray, Festara (Bere bertso guzien bilduma). A. Zavalaren edizioa. Auspoa, 35-36. Donostia. 1964.

18 Memorias de Joaquín Ignacio Mencos, Conde de Guendulain, 1799-1882. Institución Príncipe de Viana, 1952,194.
} 
Francisco Montesen ${ }^{19}$ azaina bildurgarriak, eta zeinen irudia honelaxe ematen digun euskal poetak:

\section{Hasia ilia urdintzen \\ Baina ez zangua pisatzen, \\ Bezti galtza labur ferdez, \\ Sedazko gairik senduenez, \\ Aztalak xuri zirikuz, \\ Oinetan zapatak larruz, \\ Hark duen maipolisa, \\ Izan behar da belusa. \\ Errexago da ikustia \\ Ezen ez haren pintatzia.}

Lehen korridan zazpi zezenek jokatu zuten. Montesek lehen estokadaz etzanarazi zuen lehenbiziko arerioa. Bigarrenean, nola ez baitzen oso oldarkor, jendetza zakurrak! zakurrak! oihuka hasi zen, Santerak azkenean lau estokadaz hil zuelarik. Hirugarren zezena Chiclanakoak guztiz dotore eta ederki akabatu zuen txaloen artean. Laugarrenean zaldia lurrean sabela hautsirik, lehen estokadaz bota zuen Montesek. Bostgarrenean berriro zaldiak lurrean etzanik tripak kanpoan, eta banderilak ezarri zituenean usoak atera ziren txorimalotik zezenak hau adarkatu zuenean, eta bigarren aldian sartzerakoan hil zuen Montesek. Seigarrenak berriro pikadoreak bota zituen, berriro Santera zezenzalearen kontra madarikazioak entzun zirelarik. Zazpigarrena eta azkena irinez betea atera zen, eta hamabi mutil errotariek, - galtza txuri, alkandora txuri eta zapia buruan loturik -, hamar mila liberaren ordainez saiatu ziren zezena botatzen, halere eskerrak azkenean toreatzailea atera zela, ezen batzuek gaizki zauriturik bukatu baitzuten.

\footnotetext{
19 Francisco Montes "Paquiro" (1805-1851), Chiclanan jaioa, igeltzero lanetan peoitzan hasia, baina txikitandik zezenetako grinaz amorratua, pala ezpataz trukatu zuen. Jadanik 1830.ean Sevillan toreatu zuen, eta bertako zezenlarien arte eta ofizioaz jabetu. Madriden I831.ean presentatu zen Juan Jimenez "El Morenillo" eta Manuel Romerorekin. "Garrocha"z jauzi ederrak egiten zekien, eta kaparekin xit abila izaki Espainiako lehen ezpata izatera iritsi zen. Euskal Herrian maiz ibilia, bertako koadrilak lagun zituela. Komisio batek eskatu zion Louis-Philippe, Frantziako erregearen semeentzat, Aumale eta Nemourseko dukeenizat toreatu zezan. Hasieran ezezkoa eman bazuen ere, erreginaren nahia zela adierazi ziotenean, baiezkoa eman zuen. El Fandango egunkariak honela deskribatu zuen "Korrida" : Don Francisco Montes estuvo admirable en las corridas de Pamplona. Y si es cierto lo que hemos oido decir a personas fidedignas, de que en consecuencia de su arrojo y habilidad se le ha nombrado conde de Chiclana, tiene mucho mejor ganado su fítulo que otras excelencias de escotillón (José María Cossío, Los Toros, T. III, Espasa, Madrid 1980, 632).
} 
Korrida arratsaldeko hiruretan bukatu zen, eta bostetan berriro itzuli ziren printzeak atseden zertxobait hartu ondoren. Zaldun mordo bat atera zen. beren zaldi dotoretan Luis XV eta Felipe IV. garaiko jantziez, eta bukatutakoan antzezkizuna erregina Isabel IIak parte hartzaileen artean dominak hedatu zituen. Besta hau zazpiretan amaitu zen.

Bigarren korrida irailaren zazpian ospatu zen. Erregina ordubiak pasata iritsi zen, idazkariak zilarrezko platera batean eskaini zizkion giltzak alkateari, eta honek erreginari, zeinak halaberetsu eskuinean eserita zegoen Nemourseko dukesari, beronek plazara jaurti zezan. Zeremoni hau egindakoan korrida hasi zen.

Lehen zezenak zaldien artean triskantza izugarria egin zuen. Montes, dirudienez, ez zegoen forman eta hiltzera sartu zenean jendetza aspertu egin zuen eta txistuak entzun behar izan zituen. Beste zezenak aski oldarkor izan ziren eta txaloak errezibitu zituen. Bostgarrenari hiru gizonek zaintzen. zituzten bost zakurtzar bota zizkioten, eta zakurrek zezena kozkatzen zuten bitartean, ezpata sartu zioten. Zakur hauek Jose Joakin Arrese Tolosarrak eramanak ziren bi mila eta zortzirehun errealen truke. Ikuskizun krudel eta odoltsu hau, ordea, ez zen izan ikuslegoaren gustokoa.

Ekintza hau Iñaki Azkunek ${ }^{20}$ beste era honetan biltzen eta itzultzen. digu, El Heraldo Madrid- etik harturik :

Lehen hiru zakurrek ez zuten horzka egiterik lortu. Gero zezenari bota zizkioten beste biak, hain txikiak zirenez gero, ez ziren zaunka egitera ere ausartu. Joaldunak atera eta orduerdia baino gehiago behar izan zuten zezena plazatik eramateko. Errotariek zezen hura eskatu zieten orduan agintariei biharamunean lantzaz hiltzeko. 24 errotarik beren haga punta zorrotzak lerrokaturik ipintzen zizkioten zezenari, eta zezenak hirutan hautsi zien lerroa, mutik-gazteak lurrera bota eta zapalduz.

Printze eta Printzesa frantsesek Montes zezenlariari eta bere pikatzaile Joakin Coyto, alias Charpari diamantezko eraztun bat eta esmeraldez hornitu orratz bat oparitu zieten.

Dena den, ez zuen utzi gure poemagilea ikuskizun krudel honek oso kontent eta honako bertsoa ere idatzi zuen : ni banintz erregetarik/ez liteke zezenarik. [124 b.]

20 Iñaki Azkune, Zezenak Euskal Herrian, gure baitan dituzten erro luze-ezkutuak. UEU, Bilbo, 1989. 349. 
Baleztenak 21 topaketa hauek komentatzerakoan honako iruzkin maltzurra eransten du :

Egiaz, Nemours-eko dukesa ederrak zezenari ezarri zizkioten suzko banderilak Frantziako Printzeen bidaia Nafarroarekin eta Probintziekin Foruen arazoa konpontzea eta Isabel IIaren ezkontza Carlos VIa, Montemolingo dukearekin, zeinen alde hainbeste lan egin zuten Balmes filosofo ospetsuak eta La Esperanza gazetak, helburu zuela uste zutenek hartu zituzten.

Poema euskaldunei eskaini lauko batez amaitzen da :

Irailaren 8 an goizeko zortzietan irten ziren printze frantsesak hiritik, hiru mila liberako oparia eman zietelarik bertako benefizentziazko etxeei.

\section{Eskalduna Pirenetan \\ Ez da izitu gerletan, Aitak ehortzi tokian Izanen bethi agian!}

Aski. Amai dezadan aurkezpentxo hau esanez Hiribarren zezen-korridetan oso aditua ez bazen ere bertsogile aparte zela, erritmoaren zentzu berezi eta nabarmenaren jabe, garaikideen artean poeta handitzat kontsideratua, baina halere bertsolariengandik aski hurbil dagoena, batipat hiperbatonak eta elipsiak baztertzen dituenean. Hau dela eta Lafitterentzat gertuago legoke koplariengandik olerkariengandik baino, eta bere burua epikotzat jotzen eta halakoxe nahi bazuen ere, ezin uka daiteke anitzetan moldatzen dituen bertsoak errexegiak ez direnik. Bere benetazko izpiritu zorrotz eta luma landua, ordea, ziri bertsotan, giza kritikazkoetan hala nola deskripzioetan, goi mailako eta arnas handiko ageri zaigularik. 22

\footnotetext{
${ }^{21}$ Baleztena. "Iruñerias. Las banderillas de Fuego", Diario de Navarra. 1950-IIL-25, in J. M. Iribarten. Pomplema y los viajeros de otros siglos. Pamplona, Príncipe de Viana, 1952, 170.

22 Anfon Abbadiaren koplarien guduak. Bertso era aire zenbaiten bilduma Patxi Urkizuren edizioa. Eusko Ikaskuntza-Euskaltzaindia, Donostia 1997, 89-91, 96-98, 119, 130, 156-157. 181. 182, 192. 193.197. 203, 204.
} 


\section{IRUÑEKO BESTAK 1845}

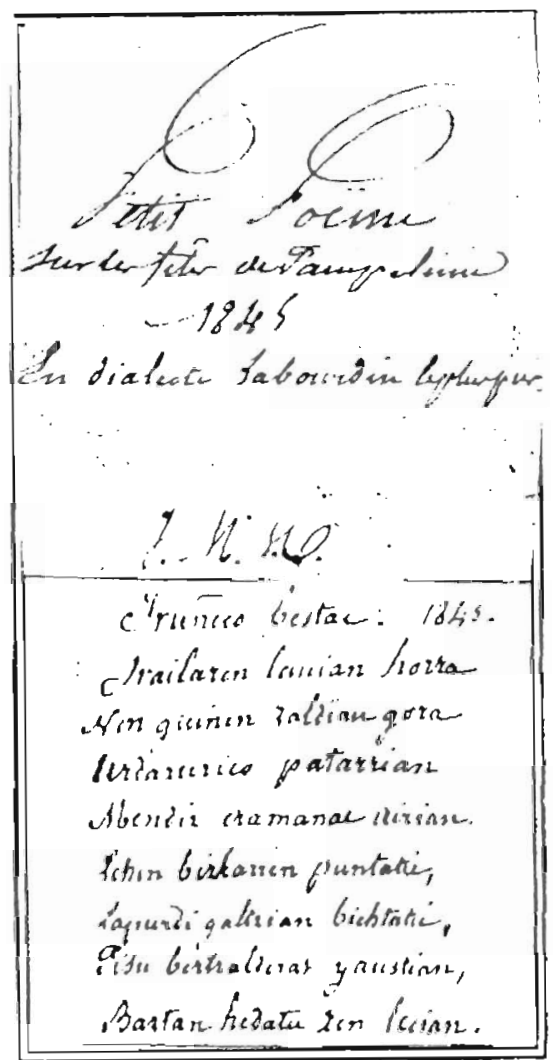

Eskuzkribuaren hasiera 


\section{IRUÑEKO BESTAK 1845}

Izan haiz, Chaho ', Iruñian,

Iraila zenian lauian.

Bada zer dukan han kaustu 2

Zertako ez duk kondatu?

Egotu nauk beira gosia

Noiz, egorriz behin asia,

Hik, Ariel ${ }^{3}$ aberatsa

Ezkiltzen ${ }^{4}$ huen hatsa.

Ah! ez dakik bada othe

Jendek zenbat hauten maite,

Jainkua utziz elizekin,

Mintzo bahiz gizonekin?

Paristarren koplak utziz

Hitan, baduk bethi untziz,

Eltze ustelian iraki,

Izpiritua ausarki 5 .

Paristarrak gatzik eztik,

Emaguk beraz hiretik.

Errak : gazen Iruiñerat, Hango besten ikusterat.

Bigarren ${ }^{6}$ egunian horra

Non ginen zaldian gora,

Urdazuriko patarrian

Mendiz eramanak airian.

1

Irailaren lauian horra

Non ginen zaldian gora
Urdazuriko ${ }^{7}$ patarrian

Mendiz eramanak airian.

Lehen bizkarren puntatik, Lapurdi galtzian bixtatik, Pisu bertzalderat jaustian,

Baztan hedatu zen lezian

Han Moya ${ }^{8}$ eta Arizkun

Urak elkharrekin lagun, Ikusten dire herrunan

Sorroz ${ }^{9}$ ferdatzen errekan ;

Etxe hazkarrekin naski,

Bainan beltzak ere aski;

Xutik utzi behar dire

Gustu zaharrian baitire.

3

Arthaldiak bi alderditan

Alha dire mendietan;

Inguru nasaiki sasi,

Hetan otsua bethi nausi;

Idatze ${ }^{10}$, othe, erreka,

Non nahi sortzen metaka;

Eremu hoietan doana

Bihotzian maiz da ilhuna.

\footnotetext{
${ }^{1}$ Agosti Chaho (Atharratze, 1810 - Baiona, 1857), Jean-Martin Hirihurrunun adiskidea.

2 Kausitu, aurkitu.

3 Ariel, Courrier de Vasconie, (Baiona, 1845-1852) eta beste deituraz ere ezagutua, Chahok zuzendu aldizkaria.

4 Moztu.

5 Ugari.

6 Eskuzkribuaren aldaera, Irailaren lauian horra.

7 Uidazubi, Urdax.

8 sic. Maia, Amaiur.

9 Soro.

10 Iratze, garo.
} 
4

Elizondo zelhai batian

Garbi jauntzia ikustian,

Bihotza zaizu arintzen,

Arraizia gaindik sortzen,

Iduri du dioela,

Oihian igeri dagola.

Hauzuak, beira ungi niri,

Non da herri ni iduri?

5

Landan badu artho, harbi;

Lurrak ekhartzen zer nahi;

Lagun maite ostatuko,

Errak hik ezetz, Patxiko?

Ez da eskas haragirik,

Ez zikhiro gizendurik;

Diru duna sakoletan

Ungi heltzen ostatutan.

6

Emaztiak dire jakinak

Nola atzeman arrainak,

Saltoz bere hoberenian

Jauztian hekin etxen aldian :

Sarian dituzte baratzen,

Amuz direnian trufatzen;

Harri azpitan aingira

Gizentzen trenxoinen beira.
7

Aitzinat, haurrak, aitzinat;

Laster Maringo zubirat,

Zubi ozar eta, haltua :

Ez egin behiti saltua.

Bi menditan ukiz zangoz,

Nonbait da erdian hondoz;

Berri-berritan egina,

Segurian dua beldur dena.

8

Barnia sudun edariaz

Ukiz izari handiaz,

Loth arimaz Belateri,

Higan mihia ageri,

Higan, higan, bethi higan,

Makhur, zutik, itzulikan,

Oihanak handi orotan,

Fagoz dire patar hartan.

9

Baldin denboraz Belaten"

Zenbat herio egin den

Nihor kantaz hasten bada,

Gorraren egitia on da :

Aitzian mendi horren leziak

Lehen gorde heriotziak,

Bada lazteko beldurrez

Eta urtzeko nigarrez.

\footnotetext{
11 Belateko Lapurrak kanta famatuaren 11. bertsoak honelaxe dio, hain zuzen :

Ainberze beldurrekin jendiak Belaten.

Uste dut pasajeruak gusto artzen duten.

Berzeren ontasunak ari ziren jaten.

Pesalunbria baizik etzuten ematen,

Pepe deskantsu ona paratu du aurten.
}

(Cancionero Vasco, P. Donostia, ed. de J. Riezu et al, Eusko Ikaskuntza, 1994, VII, n 489, 674) 
10

Harria bezain borthitza

Izanik ere bihotza,

Ez gal denbora solasen, Nola bazine Parisen;

Mendia bezin handiak

Hemen obratu gaizkiak;

$\mathrm{Ez}$ da nihon izan lurrik

Hain odol garbiz bustirik.

11

Mendiz hiru oren huntan,

Utz dezagun orai Baztan,

Erratzu ezker haranian,

Ultzama berriz eskunian;

Zuazte ahalaz airetan

Bideko bi herrixketan,

Ez baituzu gustuz segur

Janen, nahiz duzun agur.

12

Behin han jaten banago;

Hirriak bazuen gogo,

Hirugarren zerbitzuan

Ethortzian zopa kaikuan;

Garbantzotik zikhirorat,

Oliotik azetarat,

Behar zen gan hastamuka

Beldurrez nausia xoka.

\section{Argiak}

13

Iruñan gaituk, muthilak;

Bazter orotan ezkilak;

Pezak 12 pezari oihuka,

Jendia non nahi murruka 13 .

Hau da munduaren sortzia!

Gaitz da elkhar ezagutzia.

Jende orainik heldu da,

Bihar non sarthu behar da?

14

Sartzian fransesak 14 hirian,

Hiria jarri lorian,

Guziek kasik iduri

Gutan nahi aitzindari.

Lau egun hautan bederen

Ez da lanik ikusiren :

Jan, edan, nun nahi dantza;

Biba, ez diguk lan gaitza!

15

Anjelusek jo du, horra, Argiz etxetan berdura 15 :

Basuak zazpi koloretan

Izar dire paretetan;

Balkoinen burdinak suaz

lkusgarri dire gauaz;

Aingeru khoruak iduri,

Beira athen arkueri.

16

Non nahi ziren kolonak 16

Antze 17 handitan eginak,

Bere frisa ${ }^{18}$, kornixekin ${ }^{19}$,

\footnotetext{
12 Kainoiak.

1.3 Mukurru. ugari.

14 Nemours-eko eta Aumale-ko Dukien gortea.

1.5 Barazki.

16 Zutahiak.

17 Arte. trebezia.

18 Friso (gazt.), erlaizpe.

19 Cornisa (gazt.), hegaztegi.
} 
Gain-behera piztuekin;

Argiz seinalatuz kantak,

Ikusten dire paretak

Mintzo direla arraiki,

Kortesanuak bezain zinki.

17

Ez bada ere kontutan,

Komerziua agurretan

Hainitz ederki da mintzo;

Egun ona zuen atzo.

Korreiua estaltzian argiz,

Ez da errenkura astiz;

Noizbait galdu da beltzdura

Eta argitu figura.

18

Zenbat izan den fidela

Suz da mintzatzen kartzela;

Zerbitzua du oraino

Seguratzen gaixto deino.

Eliza dorren puntatik,

Ageri ziren urrundik,

Argitan distiantenak

Bafatzen 20 bere urinak 21 .

Aitu baduzu mintzatzen,

Entzun dukezu aiphatzen

Ezkila baten omena,

Hirian den handiena :

Hamabi zapatain barnian

Koka omen luzke lanian,

Elkhar uki gabetarik,

Birungan 22 denak jarririk.
20

Hartaz behiti kanpotik, Aingeruen eskuinetik, Ageri zen dorre gainian Gurutze bat ederrenian : Distiatuz ilhunbetan

Argi gaindiz gauerditan

Hedatzen zuen eguna

Guziek maite dutena.

2 I

Palazio guzietan,

Lehen plazen ezkinetan

Ehunka ziren agurrak,

Mihi zuztenak sukharrak;

Argi bat etzen ageri,

Non etzen betan koplari

Ez duke eho kalakak

Asmatua Espainolak.

\section{Zezenetako tokia}

22

Iruñan zezen lasterka

Jende biltzeko metaka,

Lehen josteta da bethi,

Unhatzen du nihor guti,

Seinalatuz geroz besta,

Lami gehiago ezta,

Denak kantaz eta oihuz

Zezenetarat hurbilduz.

23

Baldin zezenen haita da

Berant hasten ere bada,

Goizik jendia hurbil dago,

Beldurre $\ell$ gal berantago.

\footnotetext{
20 Bafatu, kolpeka argitu.

21 Esentziak, bameak.

22 Jira-biraka.
} 
Gose khentzekuaz oroituz

Plaza estaltzen da munduz, Ez plaza beharra sarri, Bainan hurbilena hari.

24

Mintzatu gabe zezenez

Dugun har idia gizonez;

Erragun non, nola diren

Hanbat jende kokaturen.

Bada pesiaz arima,

Neska, muthil, aita, ama;

Hainitz dire galtzadunak,

Ez gutiago soindunak.

\section{5}

Hoitan nihor, nahi dena,

Utz Besta bigarrena,

Bihurtu etxerat gauian,

Izanik ere beharrian;

Zaurthu ${ }^{23}$ gabe bihotz minez,

Nonbait zurrungan egonez,

Goizian goizik bazterretan

Kausitzen dire airetan.

26

Etxe bat, zabal inguruz,

Arrundan josia buruz,

Bada Takoneraz goiti,

Athe, leihuak itsu bethi.

Diot hesten dituela,

Gaitzetsi ez daiokela ${ }^{24}$,

Non ez den ankas 2.5 bestekin

Jostetan zezenekin.

\section{7}

Kanpo alderditik hori;

Dorre eztai bat iduri,

Bere zimenduz bakharrik,

Ez da gero halturarik,

Uztai etxe horren barnia,

Ez dian estalki gabia,

Mintzo naiz erdiaz, zeren

Bera leihorra den jenden.

28

Erdia hutsa utzirik

Leihor da bazterretik, Bi metra naski halturez

Athekak badire zurez, Ihes lekhu segurenak

Zezenen kontra eginak;

Hek ez balire kausitzen

Nola ager han beiratzen?

29

Harat gizonen ondotik

Sartzen direnian hargatik

Zezen errabiatuak,

Badire bertze lekhuak, Lehen athekaren aldian, Hiru urratsñoren bidian, Ezin higanak saltoka, Gutiago potikoka26. 30

Azken atheka gainetik Jar tokiak haitz onetik, Hasten dire gibelatuz, Dorriari berari lothuz.

Han dituzu inguruan

Hamabortz alkhi tornuan,

\footnotetext{
23 Zauritu

24 Dakiola.

25 Enkas, agian.

26 Lau zangotan
} 
Han dago populu xehia, Sorkhan ${ }^{27}$, oihuz ezin asia.

31

Ez du beldur iguzkia,

llez ere da jauntzia;

Gogorra delarik mandua,

Ez da hura bezin sendua;

Gizonek kaskua ageri,

Arabak buruz iduri,

Emaztiak aldian sanoki,

Kontu gabe beruak uki.

\section{2}

Zahatua han bethi mentan 28

Kurri dabil ezpainetan;

Urrixak eta ohotsak 29

Arin dauzka nafar hatsak.

Denak tripa komunekin,

Ez hargatik hutsenekin;

Zintzurrak eta mihiak

Iduri harri-biziak.

33

Haro pixgarri herrunkan,

Kantarua bethi hurrupan;

Bipela ere ausarki,

Xurienetik ez naski.

Hango jendiak, alegera,

Baden nihor hartaz gora

Ez du kasurik egiten,

Ez behin ere sobresten.
34

Han ez da khario solas,

Bokhata ere ez eskas,

Nahiz titxuak titxuarekin

Duazin bethi gatzarekin;

Karanbak eta karajuak,

Demonio berstelatuak,

Nahasten dire airetan

Ehun biben hedoietan.

35

Gorago zazpi lerrotan

Jarriz hobeki alkhitan

Arteko jende sosduna

Uriz ${ }^{30}$ kanpo duzu, Jauna!

Balkoinak baititu gainian,

Ez da nihor busti menian,

Gauza prezagarri dena

Denbora denian ilhauna ${ }^{3 !}$.

$$
36
$$

Kontu dio ohoriari, Errankizun ohoinari; Hartakotz doble pagatzen, Khetan dirua egortzen; Han hintzen Eyheraburu, Hirigoyen hire buru; Bientzat Bitoriano, Gizon maite bezain sano.

\footnotetext{
27 Estu-estu.

28 Batera eta bestera.

29 Eme eta harrak.

30 Euriz.

$3 !$ Ezdeus, ahul, triste, grisa.
} 
37

Han dire Eliza, herri,

Aphez, baile, botikari,

Eskribau herrixketakuak,

Ez ahantz sastre hirikuak,

Modistak bere pikuekin,

Erdi-andriak alabekin,

Maixtrua haur bat eskutik,

Mandozaina suz hatsetik.

38

Balkoinetik da hedatzen

Urriak duena pisatzen,

Paparo zuri, ederra,

Traba Diamanta bera;

Ez aipha infantzun majuak,

Duke, grandeza, hidalguak;

Lerruan: Oraa ${ }^{32}$ zaharra,

Baldes ${ }^{33}$ oraino hazkarra;

Martinez, gorphutzez arin

Izpirituz. zorrotz bezin;

Plumaia burutik Concha ${ }^{34}$,

Gazte bezin kasik beltxa;

Paria gobernadoria

Arropa guzia urria;

Pagoaga eta Lopez

Sahetsetik gakhuak urrez.

40

Hanbat izar lerruan josi

Etziren khoruan itsusi :

Gisa hartako uztairik

Othe da nihon bertzerik;
Birabira gain guzia,

Hedatuz bere grazia,

Ageri zen begietan

Diruaren gastuetan.

41

Xoragarri zen ikustia

Hango beztitzeko moldia :

Urdin, gorri, hori, xuri,

Zer etzen gainian ageri!

Ohorezki ageritan,

Aitzindari bulharretan,

Gurutziak lerruan metaka

Eta nihoiz bat banazka.

42

Hetaz kontu behar bada,

Frantses baloria guti da;

Frantses soldadu lehenak

Ez ditu hekin laurdenak,

Nahiz duen hurbilago

Gaitz ikusi gehiago :

Baditezke populutan

Jan beharrak ohoretan.

43

Utziz bixta hedatzerat

Erreginen balkoinerat,

Sobra othe da erratia

Zein eder den hango jendia :

Eginak guziak bertzek

Eta ez gure laguntzek,

Jainkuak amaren sabelian

Eman bezalako moldian.

32 Martzelino Oraa Lekunberri (Beriain. 1788 - 1851), nafar militarra. Lehen karlistada Zumalakarregiren kontrako ekintza gidatu zuen. Brigada-jenerala (1834), mariskala (1836), Filipinas uharteetako gobernadore eta kapitain-jenerala (1840). Nafarroara itzulitakoan senadore hautatu zuten (1844)

33 Jerónimo Valdés (1784-1855), jeneral espainola. Torata eta Villaringo kontea.

34 José Gutierrez de la Concha (1809-1895), militar espainola, lehen karlistadan parte hartu zuen. eta gero Esparteroren erorketan. Kubako kapitan jenerala (1850) cta Ministrarien Kontseilu-buru (1868). 
44

Garenen irakusterat

Agertzen gare mundurat;

Atzeman bada atzeman;

Ez da nihor sartzen aman;

Hastetik zare egina

Gero beharko duzuna;

Itsusiaren pulitzia

Alfer da igurikitzia.

\section{5}

Bederen espainol gorthia

Ez da itsusizko etxia :

Hara non den. Erregina,

Goizik emazte egina,

Adinarentzat pisuzko,

Arraizia khariozko;

Egia da nik ikustian

Urhatua zela bidian.

46

Haren larrua satinez,

Iliak berriz kasik urrez,

Nihoiz nihon itsusiak,

Kolore xuri-gorriak;

Eder bezin ona, omen,

Nork du aski laudaturen?

Jainkuak emana Jainkuari

Ungi duako handian.
47

Hari hurbil zen Infanta 35 , Eretu 36 beltxetan pulita;

Erresuman maitatua,

Den bezinbat bantatua 37

Aingeruak bezin ezti,

Erregen arraza bezti,

Bardin mantalin xuritan,

Graziak ziren begitan.

48

Bi aldetarik zituzten

Fransesen printziak ikusten,

Adituz : zer muthil paria,

Damurik ez dela guria!

Hurbil ziren gero aldian

Cristina segida berian,

Muñoz ${ }^{38}$, zeren zen ederra,

Haren Fernando beharra;

49

Narvaez ${ }^{39}$, kopeta gaitza, Iduri bezin borthitza, Zarco, San Carlos handiak, Jendez ere onhetsiak; Asis, erregen odola, Bezti soldado bezala, Eta hi gure Harispe ${ }^{40}$, Hango nihoren ahalke.

35 Infanta Luisa Fernanda (1832-1897), Fernando VII. aren alaba, Antoine de Monpensier kontearekin ezkondu zena.

36 Alde.

${ }^{37}$ Laudatua, alabatua.

38 Fernando Muñoz (1809-1873), Corps-eko guardia, erregina Maria Kristinaren eskoltakoa, Fernando VIlaz alargundu zenean, harekin ezkondu zena.

39 Ramón Narvaez (1800-1868). Isabel erreginaren tronu-defenditzailea eta Kontseilu-burua 1844az geroztik.

40 Jean-Isidore Harispe (1768-1855), militar behenafarra, Frantziako pare izendatua izan zen 1835ean eta Napoleon IIJak mariskala 1852an. 
50

Begi golpe bat oraino

Zezenak has arteraino :

Oi, zer jendezko baratzia!

Hori buruzko ohatzia!

Zer sederia ederra

Burutik lephua behera,

Broderia askorekin

Eginak orratzarekin!

51

Azken lerrotarik goiti

Ageri da behar guti.

Jendiek baleta Chinak

Han dituzte eros dunak;

Persak han ditu begiak

Seda artetik utziak;

Gathiak dire urre lodiz,

Ezpisatuak bekhaiztez.

Amerikako hazia

Han da hedatzen guzia;

Zilharginak badu hetan

Non irabaz kontuetan.

Zirikuz gordiak eskuak,

lliak legun aphainduak,

Eva berri, espainolak

Pobrentzat dire idolak.

\section{3}

Andre gazte, bai zaharrak,

Aire ausarki beharrak,

Seinale dire alkhitan

Bere lorez haizeketan;

Konda lephokuen koloriak,

Soin beltz, hori, more, ferdiak;

Hetaz dire ezaguizen

Airosak non diren jartzen.

\section{4}

Gainbehera, oi ausartak;

Berinazko larga-bixtak

Hari dire seinalatzen

Zein den hobeki beztitzen;

Han dire bethi ageri,

Zein bere urian igeri,

Urgulu baltuz jauntzia,

Bere aldian jelosia.

55

Azken hori bethi dena

Biziotan higuinena,

Atzaparrik gaixtuenekin

Eta bihotz erriarekin,

Zeinek gehiago agur

Hari dire gauza eder;

Bat haizekin zabaldua,

Bertzia despitaz hestua.

56

Ez da ederkien dena,

Markisaz laudatuena,

Inbidia fugiarekin

Antolatuz begiekin;

Guti daki hark barkhatzen

Norbaitek badu ixitzen :

$\mathrm{Ah}$, indiekin bizkarrian

Zer erremesia barnian!

57

Frantsesei girgileriak,

Hango urre, sederiak;

Xapelak Iruñarrentzat

Doian on besta maskatzat,

Lastuak iduri urrekin,

Traputzar batzuk sedekin.

Bego, bego begientzat

Nausi dena herrientzat. 


\section{8}

Ez bedi Franses andrerik

Banta hobeki beztirik;

Gathu zehatuak ziren

Bertzez neurtzen baziren.

Aire pisua damurik Iruñarrak duelarik;

Badu segur kortesia

Nor nahik bezin handia.

59

Jende deusik dakiena,

Mintzaritan ederrena,

Arraia da aldekuekin,

Bat egiten agurlekin,

Horra soldaduak berak,

Gardia ziren bakharrak,

Othoitzez zauden jendiari,

Sobratuz zuanian athiari.

60

Ez bertze asko bezala, Sakrenonden 41 daritela, Dixidatuz 42 kulazekin, Salbaiatuz konsinekin ${ }^{43}$.

Prudentzia segitzeko,

Ez da bethi fidatzeko

Kanpo itxura ederrei :

Fidatu behar da tzarrei?
61

Espainolak arrotzen du, Konfiantza galdetzen du;

Frantziako ezkinetan

Eskas zen Nafartarretan;

Iruñeko itzuliak

Eman ez baitu begiak,

Egonen dire aphur bat

Maita gabe elkhar hanbat.

62

Nafartarra da ondua,

Behar da ezagutua;

Bertziak bezala da gaixtua;

Ez egin hobiagoz saltua;

Haren lurrak ez du nahi,

Adiskidentzaren bahi,

Arras haurrai arin diten;

Etzaie hori ematen.

\section{Zezenak}

\section{3}

Ez badu ikusi berak,

Ez du erranen paperak

Zer den gudu izigarri

Mintzatzerat nohan hori.

Zezena mendi batekin,

Bere ahal ez deusekin,

Hau da gizona burutan,

Bien odola zurrutan.

\footnotetext{
41 Sacré nom de Dieu, juramentua, biraoa.

42 Mehatxatuz.

43 Consigne- consigna (frants. -gazt.), agindu.
} 
64

Kostuma zaharren errua!

Noiz zaik xarturen ondua?

Ordu huke suntsitzeko,

Eztiago agertzeko!

Ikusatzu atheratzen,

Elkharrekin aitzinatzen,

Hamarbat gizon tieso,

Lerruan jarriz besoz-beso.

Hartzen dutena erdian,

Mentadun herri guzian,

Duzu erregen aiskidia,

Montes, josteten hastalia.

Montes bere lagunekin,

Bertzez kasu gutirekin,

Badua zerbeit eskatzerat

Erregien balkoin perat.

66

Errespetu handienian

Han dago belhaun bat lurrian,

Humilki buruz behera,

Eta denak hari beira.

Gakhua, libragai zezena,

Aski iguriki dena,

Arthikitzen zaio airez,

Moldatua erribanez.

67

Nemurseren andriak bada,

Isabelen nahia da;

Izan du ohore hori,

Beiratua lehenari;

Errege espainolentzat

Agertzen bethi nausitzat;

Zezenetan berian ere
Hortako frogak badire.

68

Aphez eta mirikua,

Pare bardin ohitua,

Athian dituzte ezartzen,

Eian nihor den behartzen.

Paperez eginik gizon bat

Ethortzian plaza erdirat,

Hirriak dire saltatzen,

Ez baitu nihor izitzen

69

Lehen barrera athetik,

Bigarrenaren azpitik,

llkhiz xahalen aita,

Sartzen da plazan saltota;

Patroina bakharri kaustuz,

Hasten zaio sudur ziztuz;

Mundua oihuz goratik,

Mila biba ingurutik.

70

Laster ikusteko zer den,

Hartuz boliada lehen,

Hor tripatik adarretan

Ematen du girginetan;

Patroinak tripa bultua

Ihiziz zuen hantua;

Hirriz eman zen mundua,

Xoriz bethetzian zerua.

71

Han uso bat plazerekin

Ikusi zen hegalekin,

Ez jakinez jautsi norat,

Gaten erregina ganat 44 .

Lehen Erroman sēgurki,

Iruñian bezin goraki,

\footnotetext{
44 Hona nola kontatzen duen kronikagile erdaldunak: “...banderillas de las que salen pájaros y palomas, tres de éstas se posan delante de la reina quien las coge. acariciándolas el duque y duquesa de Nemours, mientras su Magestad ordena conservar los pichones con cuididdo para transportarlos a Madrid".
} 
Banaturen zuten hola.

Zerua mintzatzen zela :

72

Isabela, zure khorua!

Jabal ${ }^{45}$ bedi Carlos 46 berua;

Sorthu zare erregina;

Ixil zeruko dohaina.

Erreginak Nemursari, Eman zion uso hori :

Hartaz artha izan badu, Zuina higatzen du!

Aski da jostetarena;

Bilha begiez zezena.

Burua kasik zeruan,

Arrapua suilaz musuan,

Behatzez joka lurrari,

Marrumaz beira jendiari,

Plazan dabila lasterka,

Desafiatuz adarka.

74

Nor da bada agerturen

Orai kontra zezenaren?

Ausartzia handirekin

Montes bere lagunekin.

Zein den gero jakiteko

Hemen dut seinalatuko;

Ez da ederrenetarik,

Ez eta itsusitarik.

75

Hasia ilia urdintzen,

Bainan ez zangua pisatzen;

Bezti galtza labur ferdez,

Sedazko gairik senduenez;

Aztalak xuri zirikuz,

Oinetan zapatak larruz;
Hark duen maipolisa

Izan behar da belusa.

76

Errexago da ikustia,

Ezen ez haren pintatzia,

Dirdiratuz iguzkian

Ehun izarrez begian,

Zilharra xoilki ageri,

Zertako beira zolari?

Maipolis barnekotik

Paileta gandi sedatik.

77

Urthez dohanian bezan bat,

Badu berroitahamar bat;

Kortesia denak baitu;

Tretu bizi, beltxak ditu;

Ez da bizkarrian kargarik,

Ez lasterkaren trabarik;

Bururaino espanta,

Ezagun du zaila dela.

78

Hark bizkar xuriz zuena,

Lagunak horiz emana,

Zen galtza motx arrosekin

Ez gutiago senduekin

Besuan zituzten garbiki,

Doblian behiti puliki,

Kapa batzuek arinak,

Hori, urdinez eginak.

79

Sare beltz sedaz buruak

Ezin hobeki lothuak,

Erribanez ${ }^{47}$ katagona

Lephuan behera zohana.

Hek bezala beztitzeko

Behar da zenbeit untzako.

45 Baretu.

46 Carlos V, karlisten erregea (1788-1855), ez dirudi Hiribarrenen begiko zenik.

17 Zintez. 
Holakuak toreadoriak, Zaldiz dire Pikadoriak.

80

Azken hoiek zezenetan

Nola nobliak denboretan,

Xapel zuri zabalekin,

Beztidura horiekin,

Galtza hazkarrenak larruz,

Barrutik bardin xabalduz;

Haga luze bat eskuan,

Zorrotz haltzairuz buruan.

81

Zaldia begiak gordia,

Inguru mokanes ${ }^{48}$ ferdia.

Hekien ondotik txuluak,

Ez baitire behar tontuak,

Bezti, iduri dutela

Figaro ${ }^{49}$ piztu zaiela

Bere jauntzi guziekin

Tirriatzeko heiekin.

82

Jo du trunpeta airosak;

Huna gudu seriosak;

Ixil jartzen da mundua,

Nola balitz izitua :

Plazan zaldiz pikadoriak,

Eskutan haga traidoriak,

Hurbil ilkhi tokiari

Beira daude zezenari.

83

Xahalaren aita gaitza,

Farrastan lurrian behatza,

Erro zabilan inguru,

Ez jakinez nori buru,

Izitua, nahasia,
Ahua arrapoz gaindia,

Jendiaren harramentzekin

Errabia guziekin.

84

Izan gabe flakatua

Asma guzia galdua

Bazterreko ihurtzuriz,

Egorriak aho goriz;

Zanguak, burua, buztana

A.lferretan zabilkana.

lkus duen bezin laster

Bultu bat inguru ezker.

85

Hor duako hiru saltotan

Jastatzeko adarretan;

Frixtan ${ }^{50}$ hurbildurik kolpez,

Miran hartzen du berustez

Pikadoriaren zaldia,

Barrera kontra ilkhia;

Zaldi bakhotxa zaurituz,

Lasterka dua aitzinatuz.

86

Zaldikuak luzatuz lantza,

Sistatzian espalda gaitza,

Baldin ungi hartzen badu

Hainitz aldiz izitzen du;

Bera bada izitua,

Mundua ere laztua

lkusiz, odolian denak,

Zaldi bulhar hazkarrenak.

$$
87
$$

Oi, nola iliak buruan

Xutik diren inguruan,

Beldurrez odola hila,

Hatsik, ez hitzik ez dela!

\footnotetext{
48 Musu-zapi.

49 Figaro, Beaumarchais-ek asmatu pertsonaia, Le Barbier de Sevilla obran (1775). Mozartek eta Rossinik erabili zutena beren operetan : Le Nozze di Figaro (1786), eta /l Barbero de Sevilla (1826).

50 Maiz.
} 
Biziaren estalkia,

Gainekuari adrezia

Orduan eskasten bada,

Lazdura sobranitan da.

88

Lurrerat dua erroz gora,

Zaldia arin gainera.

Ouf! Bera ez da ukia;

Bainan nola den zaldia!

Bulhar guzia ageri,

Sahetsetik odol gorri;

Tripa dario herrestan

Zezenaren ohoretan.

Zaldizkua, segur iduri

Zeren dituen ezarri

Burdin xabalak non nahi

Kolpe gaixtuen urrun bahi, Fundis! lezake zezenak

Urrun balitu lagunak;

Bainan hurbil dire txuluak, Arin dituztenak zanguak.

90

Ezpadak hekin buruan, Airetan harmak eskuan, Zezenari dire gaten, Jauziz orotarik hasten; Sobra hurbil gabetarik, Bere kapak arthikirik, Zezena dute zoratzen, Haren bitima salbatzen.

\section{1}

Hanbat kolorez deithua, Zezen errabiatua, Heki duakote lauhazka, Utziz zaldia ihauska ${ }^{52}$.

Zer ophila bihotzetik

Dena urruntzen geroztik!

Heriuari hurbilena

Da geroztik segurena.

92

Arin behar da orduan, Kontu egin inguruan; Norat behar den ihestu Aitzinetik begiztatu, Toleadoren ondotik, Ahurina ${ }^{53}$ muthurretik, Dualakotz airez zezena, Guzia odol egina.

$$
93
$$

Atheka behar da geroz Bien artian eman saltoz, Beiratu gabe gibelat Etsaiaren jostatzerat. Arthikitzen zaio kapa, Nahiz ethortzian lasterka, Jabalarazi gezurrez, Tentatzaliaren itxurez. 94

Maiz ikusten da hargatik Aitzinatzen kapagatik, Atheka jauziz pasatzen, Ikharaz denak bethetzen. Asko aldiz pikadoria, Erroz gora zaldiz gordia, Zezena hurbil duenian, Ez da mintzo haren menian.

\footnotetext{
51 Hondatu, desegin.

52 Zilipurdika.

53 Adurra, lerdea.
} 
95

Miletan dohatsu baldin

Ez badu jo adarrekin!

Haren musuko ferekak

Ez dire amen besarkak;

Itziliphurdi egitian

Loth baditeke barrerian,

Lagunek hartuz barnerat

Hel diteke segurerat.

\section{6}

Zenbat aldiz dago ordian,

Ubel, umatua, lurrian,

Barreraren kontra zapan,

Odola gaiaz zurrutan,

Zezenak su handienian

Uki-beldur buru fuinian;

Ez badiote urruntzen,

Laster heriua agertzen.

97

Xarpak ${ }^{54}$ xoilki hiruetan,

Zaldia on izlapetan,

Eman zuen munduari

Atsegina pasagarri;

Hari hil arte azpikua

Kobarde zezena sendua;

Bainan hura galtziarekin,

Bera jarri kobardekin.

98

Batek zezena adreki

Biraka zuen puliki,

Buztan puntatik lothurik,

Han ibili zoraturik,

Zezena bira bezanbat

Hura lastertuz aitzinat,

Hiru erri etzelarik

Iphurdirat adarretik.
99

Zezena saltoz ethortzian,

Frixtan bazakien airian

Kaparekin enganatzen,

Haren dexiduz trufatzen

Hil denian zenbait zamari,

Aski zaldizko erori,

Besta jaunzten da berritan,

Guziek maite gustutan.

100

Utzi plaza pikadoriek

Hartu behar dute txuluek;

Urruntzen dire zaldiak,

Zangoz egiten jauziak.

Hirietako trunpetak

Eta xirola bastartak.

Oihuz daude aski dela

Zaldiz ixuri odola.

101

Metra eskas den makhila

Txuluek dute banderila,

Haltzairuzko puntarekin,

Sarthu behar lasterrekin

Zezenari lepho gainean,

Egon behar adar menian,

Gauez hari bekhoz-bekho,

Beldur gabe harendako.

102

Eian behar dute oinik,

Buru, begi, bihotz iminik!

Doblezka dire makhilak

Fida direnian muthilak;

Makhila hek ingurutan

Paper kroxkatuz jauntzitan, lkusgarri hala hala,

Zezenen lephuan bezala.

54 Xarpa pikadoria, zeini frantses printzeek diamantez hornituriko eraztuna oparitu zioten. 
103

Bethia dire petartekin,

Zeineri khardo mitxekin5s

Baitzaie sua ematen,

Etxa ${ }^{56}$ diren baino lehen;

Folsua ungi dagola

Sartzen denian banderila,

Ethorriz aitzin aldetik,

Ginatuz adar artetik;

104

Bertzenaz da ikustekua

Banderila ondikozkua,

Arthikia adarrekin,

Gor ahaleko bestekin.

Pikoz eta suz mindua,

Zuziez irakitua,

Zezena saltoka orruaz

Jartzen da gora buruaz;

105

Espaldetako zauriak,

Doble berak idekiak,

Ero dabil jo lasterrez,

Mendeku nonbait beharrez,

Ozartu nahi delarik,

Indar guziak bildurik,

Errabiak hazkartua

Nahiz hainitz izitua.

$$
106
$$

Bainan harentzat azkena

Jo du trunpeta orena.

Eskuz muleta hedatuz,

Beira nor duan aitzinatuz,

Nola errege zetruari,

Lothuz arrai ezpatari,

Txulozko lerro batekin

Bihotzeko bakiarekin.
107

Deitzen da hori ezpada,

Holakoez paria bada;

Ez duen gisa parerik,

Hortan Montes ageririk.

Aphuru lazgarri dena,

Ezpada eta Zezena,

Bekhoz bekho dire jartzen,

Elkharren indar miratzen.

108

Zezen aitzinian gizona

Behar izan artexena,

Hura ondartzen zaionian

Jartzeko adar gerizian;

Zezena han da ezarria,

Nahiz ase errabia,

Ezin gorde dezakena,

Odol galdetzen duena.

109

Ezpadaren banderari

Zezena da beira jarri;

Higuin ahal da gorria;

Hark eman dion furia!

Hantu da hainitz lephotik.

Hura ikusiz geroztik;

Zartatzerat duazko zainak

Bere indarren pikainak.

110

Nola orduan gizona

Diteke galtzetan barna!

Ez du ageri kanpora,

Bainan du asko pirpira.

lkuspen horri josiak

Daude guzien begiak;

Ez da deus bertze ikusten,

Nahiz asko den amesten.

55 Metxa, sugailua.

56 Bota. 


\section{1}

Noizere bada zezena,

Gehiago ez daukena,

Baitua saltoz gizonari,

Ez ustez segur bertzeri,

Horra, ustez jo etsaia,

Non duen atzeman zaia,

Beso ezker petik airian

Hiltzaliak bide egitian.

112

Eskuinaz dio adreki,

Buru galdu gabe, finiski,

Ezpata hezur artetik.

Zuzen sartzen garrotetik,

Bi alde espaldan uzten,

Lehen kolpe lurrian ematen,

Gorphutz guzia dardaran,

Azken biziko pirpiran.

113

Harma atheraz frixtakuan

Zezenari hiltzerakuan,

Altxatzen du balentriaz,

Agurrarazten bilkhuiaz.

Bere du lehen zezena,

Urre balio duena,

Gortheko heian hazia,

Gosta delakotz bizia.

\section{4}

Badu oraino harekin,

Aberatsen agurrekin,

Asko gauza, zilhar, urre,

Sederia eta lore;

Guzien mihi puntetan

Haren izena lerrotan,

Sekulakotz da ikhasten

Ohoriak zaizko ematen.
115

Bainan zezena ukia;

Aldian utziz hil tokia,

Gaten bada sarraskitan

Odola gaiaz zurrutan,

Haragiz lephua ezagun

Kolpatzalia ganik urrun,

Molde gabiaren paguak

Dire trufazko xiztuak.

116

Zezena bada nausitzen

Eta gizona izitzen,

Bapo da xahalen aita;

Bapo gizona hileta!

Ezpada hilik bestetan

Deus ez da eskas jostetan;

Bibak dire bazterretik,

Egorriak bihotzetik.

117

Ezpada gudutik denian

ilkhitzen trionfa menian,

Ikusten da hainitzetan

Kortesiazko pausutan,

Andre handien zenbaiti

Xingola butatzen goiti :

Heiek dute seinalatzen

Hila nondik den ilkhitzen.

118

Nork erran galanteriak

Hor zituela "graziak"?

Moruek dute Espainian

Hori utzi zahar mendian.

Hiru egunen segidan

Plazak odola du edan.

Ehun zamari bederen

Ezpatan pasatu ziren. 
119

Hekin hertzezko berdurak

Galdetzen zituzken urak;

Bainan ohituak naski

Nihork etzuen arthiki;

Zezen bat hiltzen zutenian

Hiru mandok ederrenian,

Lepho kraskailaz bethekin

Zaramaten lasterrekin.

120

Gizonak unhatu onduan, Eguna jabaltzerakuan, Zakhurrak ziren largatu, Zezenekin engaiatu;

Etzuten eman amorrik

Nahiz etzuten adarrik;

Hainitz gathasken buruan

Nausitu ziren guduan.

121

Zezenak zituen behin

Butatzen airetan arin, Hartuz adarren puntetan

Hurbil aldi guztietan;

Bainan zirenian oroitu,

Batzuk beharritik lothu,

Bertziak ixter zainetarik,

Zezenagan eskutarik.

122

Hiltzeko azken zezena,

Bildu zen errotazaina;

Hamabi ziren hetarik,

Denak xuriz beztiturik,

lliak irineztatuak,

Batzuk bertzeri lothuak,

Batian zuazin elkharrekin

Haga luze batzuekin.
123

Jainkuak beira makhurtzetik, Lagunen lerrua uztetik!

Bakhar denak berehala

Eginen du karanbola :

lkusi ziren nigarrak

Zezena sarthuz adarrak

Bi muthilei ziotenian

Hatsa khendu hoberenian.

124

Hainitz gathasken ondotik,

Hamabi lantzen sasitik,

Lotzen dute paretari

Onduan lotzeko lurrari :

Utz ordu dire zezenak,

Espainolentzat eginak:

Ni banintz erregetarik,

Ez liteke zezenarik.

125

Horrek ez du espainola

izituren berehala;

Ez dut hekien gusturik,

Halere naski nererik.

Dena den, adio bethikotz

Etzarete neretakotz,

Josteta hirriskutsuak,

Barbarian asmatuak! 


\section{Zaldi Lasterkak}

\section{6}

Plazan sarthu dire betan

Hogoi muthil zamaritan;

Bizarrak doian zioten

Kokotsian itzal egiten;

Gorputzez ziren lerdenak,

Larruz itzalian egonak;

Har zitezken jauntientzat

Iguzkiko erregetzat.

Bat zen bezti lander hariz, Kapatik hasirik xuriz;

Buruan estalki kobria, lduri baitzuen urria; Kaskatik lumek airetan, Haiziaren hatsez jostetan, Edertzen zuten nausia, Jadanik ez itsusia.

Belusa zen bertzia jauntzi ; Halako sedekin untzi Baliteke bilhatzeko; Deus hain pulitik gaineko, Kapa labur bat ezkerrian, Akhabo dena belhaunian. Gaphelu xabal batekin Bi lumaia xurirekin.

129

Hirugarrena ez gaizki

Bezti zen estranioki, Galtza largo batzuekin, Morotako pikurekin, Sabre eder bat dilindan, Oihal gaphelu kopetan; Sedaz bordura guziak, Kapusailian urreriak.
130

Nihor etzen bezti hetan, Bat bertziaren arropetan; Loria bat zen ikustia, Ez aipha hala izatia Urrezko estriberekin, Lantza punta zilharrekin, Zangotako brodekinak Arropen funtsian eginak.

\section{1}

Zaldi ezin itxikiak Emanak Andaluziak, Bere gainekuez hartuak, Lasterkarat paratuak, Farrastan lurrari joka Nahiz eman galupaka, Ahotik zirrapua jari, Irrintzirinaz mintzari.

132

Galdetzen dute lasterka Abiatzia zein lehenka, Ekharriak lehertzerat, Ahal guzien urtzerat, Bere nausiak trionfan Eremateko airetan, Iduri badakitela Bertzenaz deus ez direla.

133

Airetan zen ereztuna Behiti hariz emana. Zaldizkuek bere lantzekin, Zuazilarik lau hatzekin, Behar zutena zilhotik Atzeman lehen kolpetik; Behar zen besta hortako Begi on eta zaldizko. 
134

Zaldizko bakhotxak bada,

Hartuz geroz boliada,

Segura zuen berekin

Ereztuna xingolekin;

Segitzen zaion xingola,

Kometa buztan bezala,

Lantza punta zuenari

Sarthu ereztuen zilhuari.

135

Zaldiak zuazin airetan

Largatuak bridanetan,

Lurra seinale etzela

Zangoz ukitzen zutela, Hekin gainean egoteko,

Gehienentzat lan asko;

Bainan hagan zituztenak

Etziren beldur zutenak.

136

Lasterka horren ondotik,

Sega baten ingurutik,

Atxatzen zituzten loriak,

Zaurthu gabe beso luzeak;

Hoitan ere gehienak

Izan ziren eskaldunak :

Erran nahi da artexak,

Diona hitz horren funtsak.
137

Moro bat eman zutenian, Xutik alkhi baten gainian, Zaldizkuak bazuazun hari, Bulharrak zaurthuz pekuari, Bainan etzaizkon hurbiltzen, Zaldiak ziren izitzen, Behin abiatu orduan Gibelat egiten saltuan; Xanxak han onduan ziren; Zanpelei lothu behar zen 57 . 138

Batek zaurthu zuen Morua

Eta irabazi khorua,

Ezin hurbilduz nihondik

Lantza arthikiz urrundik.

Norbera baita suntsitu

Lantzak duena zilhatu, Bestak dire ganbiatzen, Berritan emanik sortzen.

139

Zaldizkuak bi burutarik, Arroltze z zarpak betherik, Hor dire hasten elkharri Besainka, haurrak iduri; $\mathrm{Ez}$ da zilegi gelditzia, Zaldi galupa baratzia;

Besainka zarpak huts arte, Gutik amor eman dute.

140

Kobrezko eskudak zuzten

Beso ezkerrian ematen, Bisaiatik beiratzeko

Kolpe hori arroltzezko; Hetan leherturik kroskuak Saltatzen ziren gorringuak; Kiski-kaska zuan denbora, Ekhia sartzian ohera. 
Zuziak

\section{1}

Plaza nausia arratsian

Ezin agertu zen hutsian, Estali zuten arbolaz,

Hekien gainak bolboraz, Paperezko adarrekin, Plegatuak uztaiekin, Asur arrudak iduri, Edo zenbait asmu berri. 142

Bi oren handiz bederen

Etzinduen ikusiren

Plaza guzian pixturik,

Bertze nihongo argirik, Iduri plazer zuela, Besta eder zitzaiola, Zeruak berak izarrak Utzirik pixtu beharrak. 143

Arrudak dire birungan, Ginharrak denek burrunban, Zuzirien ithurriak, Izarrezko zurxuriak; Argiz eginik dantelak, Hedatuak bere belak, Argi arte denetarik Deseinuak agerturik.

$$
144
$$

Orga, gaztelu, arkada, Iguzki, lanpa parada, Zer nahi den figura zen Kartuxatik atheratzen; Gaten zirenak airetan, Jauzten ziren urgulutan Ehun arraioz gaindiak, Kolore urdin-xuriak.
145

Plaza Castillo erdian

Gauza probetxos berian, Ithurri bat ur onekin Ikusten da plazerekin, Hura ere paretetan, Dena bezti kartuxetan, Aphaindua zen gerlako, Su luzeki egiteko. 146

Hiru gerlako fregatek, Higituak mekanikek, Jautsiz gain behera betan, Gordiak zuzten arrudetan, Hiru aldetarik belan Eman zioten asko lan; Hara hunat bazabiltzan, Nola itsasoz balentzan.

147

Guzietarik deskarga Betan egiten aldizka, Biratzeko artexketan Balak igorriz panpetan; Bonbak ziren iguzkiak, Balak izarren pesiak; Fortiak amor eman zuen Zuzirik noiz ez baitzuen. 148

Pulunpatzen ziren ganian, Kolpez aurkhitzen egunian; Zenbatenaz ilhunago, Hanbat orduan argiago, Oi, zer gau ederra hura; Ez da halako ardura, Ez eta ere beharrik, Inguru denian beharrik. 


\section{Zuziazko Tiruak}

149

Hamar orenek jo onduan, Hedatua jendia heguan Beira zagon denborari, Zeru garbi, ederrari, Zitadelan karrosekin, Gibeletik oinezkuekin, Sarthu zen gorthe guzia Argiz zuela hesia.

150

Kanpotik eta barnetik

Alderdi guzietarik, Birabira harrasiak Zuzko sokekin argiak, Bastun hegi guzitan, Inguruko itzulitan, Hiru herrunka bederen

Suz piztu basuak baziren.

\section{1}

Portaliak gain beheretan, Zubien bere gathetan, Denak suzko argiekin Arin ziren gauarekin; Ezker eskuin hainitz urrun, Milaka bazen lanpiun, Ezarriak halturetan, Bide bazter guzietan. 152

Hetaz piztuak arbolak, Hetaz gaztela kantaiak; Etzen uste bizi lurrian Bainan ametsezko gauian, Ethorriz zitadelatik Argi nola eguzkitik. Agertu zen zitadelan Tronu bat egina gelan;
Zariola sederia, Franjetan dena horia, Arkajuz egin colonak, Urria gaindiz daritenak. Gorthe dena han zen jarri Beira zuzien gerlari; Sua zen hasi kanpotik, Soldaduz zen larrainetik. 154

Balazo enganatzaliak, Ederrenian beztiz airiak, Jausten ziren izarretan Ibiliz nola jostetan; Pezak ixil hari ziren Arthikitzen iguzkiren; Oren batez zeru dena Izan zen sutan emana. 155

Bazen kasik errateko Sobera zela lurreko; Halako gerla ikustia Nihorentzat nigar bidia; Soldaduentzat odola; Bainan kontu diotela Kekin ezpata gorriak Seinalatzian justiziak. 


\section{Pasaiuak}

156

Ilhun alderat hirian,

Pasaiatuz agerian,

Gaindiz da jendia ikusten, Ttipi handiak nahasten.

Edergailu munstratzeko, Hara-hunat ibiltzeko, Jendiak ilkhi nahi badu, Takonerak gaitzik ez du. 157

Nahi denian norbeit jarri Ez da nihon eskas harri; Badire itzal ederrak Eginak platan adarrak, Erdian zabal baratziak Arraiki zabalduz loriak, Hala nola koloretan, Denak gaindiz usainetan.

158

Hiri barnian da pasaiua, Ederra bezin arrarua, Jendez, nola liliekin, Bethi bezti gusturekin. Hortik lekhora kafetan Jendia sartzen arratsetan, Gizonak bezala andriak, Kostuma idekiz athiak. 159

Bere bolada esnekin, Limonada arnandekin, 58 Pausu handienian husten, Pezeta erdi iristen; Tripak ederki betheta, Higual zaiote zer gasta; Jendia bizi da beroki; Gutitan naski hobeki.
160

Onhetsia alkhietan

Jartzia aphezak lerrotan, Kate, pasaio, zezenak, Guzientzat dire onak; Hetan bakhotxa beretzat, Jelosla nihorentzat :

Berak egiten duena

Nola bertzetan kondena? 161

Jeloskorra, har eskola

Ixil egon behar dela

Bertzek nola hik egitian

Bere bizitzeko moldian.

Bere ustez jakinsunak, Badire ikusten jaunak Trufatzen direnak jendez. Beztiak hekien tintez. 162

Hek, kantatuz libertatia, Hustian kafetako athia, Bidian nihor ezin jasan, Guziak ezartzen basan, Iruñen ez halakorik, Han nihork ez ahalkerik, Egitian bertzek bezala, Aphez edo lego dela.

58 Arbendolekin, almendrakin. 


\section{Harmada}

163

Ederra zen egun hetan,

Iruñeko zelhaietan,

Soldadu lerruan emana,

Francisco Asisek ${ }^{59}$ mana.

Beruak etzuen izitu

Nahiz nola zen agertu

Gerla bide itzulitan

Utziak aski berritan.

164

Jendia zagolarik erria,

Sapha minian baitzen airia,

Soldaduak arratsaldian

Kausten ziren hoberenian;

Ibili ziren lasterka,

Aitzindariak oihuka,

Goiti behiti lerrotan,

Erregen gustu handitan.

$$
165
$$

Sarthu zirenian hirian

Etzen bustirik bisaian;

Hanbat lasterken ondotik,

Doian ezagun hatsetik

Asisen zaldizkuek zuten

Sartzian ederren ematen;

Bainan bazen oinezkotan

Zain hartagorik menbrotan.

$$
166
$$

Halako soldaduekin,

Aitzindari jakinekin,

Bentzu diteke mundua,

Nahi bezin hedatua.

Ez dire beldur gosiaren,

Ez eta ere bidiaren,

Guti janik ikhasiak

Laster higaten mendiak.
167

Suntsuna, xirribikekin,

Nafartar soldaduekin, Gerian dabilke jostetan

Nola Eliza bestetan.

Goizeti arrats askotan

Suan eta mendietan,

Zigarroa bat musuan

Bizi ditezke multzuan.

168

Sos baten agorienta

Hekin edari plazenta;

Soldadu on ilkhitzeko

Bada heki beiratzeko.

Erromanuen denboretan

Lehoin ziren mendietan;

Ondoriuak bizi dire

Gutiago ez baitire.

169

Bertze hanbat jende zoro, Guziak direnian haro, Zer diren eskaldun umiak

Errateko zen, aizkidiak :

Lau mila urthe bederen

Nausi dire toki beren;

Ez da munduan bertzerik

Hek bezala itzaturik.

170

Higatu dire mendetan

Populuak bazterretan;

Bere lurretik suntsitu,

Nausiek asko kasatu,

Eskalduna Pirenetan

Ez da izitu gerletan

Aitak ehortzi tokian

Izanen bethi agian.

Aski J. M. H

59 Francisco de Asis Maria Fernando de Borbón (Aranjuez, 1822 - Epinay-sur-Seine, 1902). $1846 \mathrm{can}$ bere lehengusina Isabel IIarekin ezkonduzen 